\title{
Features of inference for abnormal truth values of premises in $\mathrm{V}^{\mathrm{TF}}$-logics
}

\author{
L V Arshinskiy ${ }^{1}$, V L Arshinskiy ${ }^{2}$, M P Dunaev ${ }^{2}$ and M S Nitezhuk ${ }^{1}$ \\ ${ }^{1}$ Irkutsk State Transport University, 15 Chernyshevskogo Street, Irkutsk, Russia \\ ${ }^{2}$ Irkutsk National Research Technical University, 83 Lermontova Street, Irkutsk, Russia \\ E-mail: larsh@mail.ru
}

\begin{abstract}
The article discusses the issues of logical inference for abnormal truth-values of premises in logics with vector semantics from the $\mathrm{V}^{\mathrm{TF}}$-logic class. Such situations as strict lies, uncertainty, and complete contradiction are considered in this article. It is shown that the truth of the conclusion in this case can take an interval value and the nature of this interval is preserved throughout the inference. This feature can be used to detect knowledge base artefacts during dynamic verification of knowledge bases.
\end{abstract}

\section{Introduction}

Among artificial intelligence systems, knowledge-based systems play an important role [1-4]. Often these systems use multivalued logics or probability theory (for example, Bayesian inference) $[3,4]$. Non-classic truth values, in addition to Truth and Falsehood, can be interpreted in different ways, for example, as the degree of confidence or belief [5-8]. They can express the modality of the necessity and possibility, reflect the semantics of possible worlds (para-consistent logics), and so on [5]. In probabilistic inference, truth is replaced by probability, which can be considered as an analog of the degree of confidence [4].

As a rule, when developing such systems, they use the truth scale $[0,1]$ or its analogues: $[-1,1]$, integer values from 0 to 10 , from 0 to 100 , from -100 to 100 , etc. At the same time, there is a problem of separation of situations when nothing is known about an object and when information about it is contradictory. Both cases represent the middle of an interval, for example 0.5 or 0 (for scales of type $[1,-1])$ and do not differ from each other.

Schaefer's evidence theory and interval confidence estimates provide broader possibilities [9]. However, certain difficulties can be found here (for example, difficulties of algorithmic nature) [3].

To avoid these difficulties, a vector truth model was proposed (see for example [10-13]). In this model the truth is described by a vector with components (aspects of truth) 〈True; False $\rangle$. Aspect values are determined by independent sets of evidence and belong to the range [0,1] (in application systems, it is convenient to use integer values, for example, from 0 to 100). This allows to formalize situation of strict truth $\langle 1 ; 0\rangle$ strict lie $\langle 0 ; 1\rangle$, uncertainty $\langle 0 ; 0\rangle$ and contradiction $\langle 1 ; 1\rangle$ of propositions. In general, the components are some numbers from the specified interval, which are determined by the degree of confidence in the received evidence. Situations of uncertainty (when there is no information) and contradictions (when incoming information contradicts each other) are naturally separated in this view. Such logics can be considered as fuzzy para-consistent logics.

Copyright (C) 2021 for this paper by its authors. Use permitted under Creative Commons License Attribution 4.0 International (CC BY 4.0). 
In [10], and later in [12], this view was generalized to logics with an arbitrary number of aspects, but $\mathrm{V}^{\mathrm{TF}}$-logics seem to be more interesting in practical terms. This approach was also developed for the interval representation of the truth vector $[11,12]$.

One of the advantages of this approach is the ability to make logical inference in conditions of abnormal truth values of premises: strict lies, uncertainty and contradiction. This could be considered as a technique for identification of hidden anomalies of knowledge bases of application intelligent systems.

First of all, those anomalies are detected dynamically during the inference.

The problem of knowledge bases anomalies detecting knowns for a long time [14-20]. In the works $[21,22]$, this question was described for "point" values of the truth of premises, when the truth vector is a point in the square (Cartesian product) $[0,1] \times[0,1]$. In contrast to previous works influence of abnormal interval truth values on the entire inference chain is discussed here.

\section{Inference at interval values of the truth}

The following inference rule is used $[11,12]$ :

$$
\begin{aligned}
& a, a \rightarrow b+b:\|b\|=\left\langle a^{+}{ }_{1} \bullet i^{+}{ }_{1} ; a^{-}{ }_{1} \oplus i^{-}{ }_{1}\right\rangle \div\left\langle a^{-}{ }_{1} \oplus i^{+}{ }_{2} ; a^{+}{ }_{1} \bullet i^{-}{ }_{2}\right\rangle= \\
&=\left\langle\left[a^{+}{ }_{1} \bullet i^{+}{ }_{1}, a^{-}{ }_{1} \oplus i^{+}{ }_{2}\right] ;\left[a^{+}{ }_{1} \bullet i^{-}{ }_{2}, a^{-}{ }_{1} \oplus i^{-}{ }_{1}\right]\right\rangle .
\end{aligned}
$$

Here $a, b$ - propositions, $\rightarrow$ is the symbol of implication, $\|b\|-$ truth of a proposition, $\langle\ldots ; \ldots\rangle-$ truth value as a vector with the corresponding component values True and False; $\langle\ldots ; \ldots\rangle \div\langle\ldots ; \ldots\rangle-$ the range of values of truth vector as the rectangle defined by top left and bottom right corner on $[0,1] \times[0,1]$; for example, truth values of small premises $a$ is $\|a\|=\left\langle a^{+}{ }_{1} ; a_{1}^{-}\right\rangle \div\left\langle a^{+}{ }_{2} ; a_{2}^{-}{ }_{2}\right\rangle$ or $\|a\|=\left\langle\left[a^{+}{ }_{1}, a^{+}{ }_{2}\right] ;\left[a_{2}^{-} ; a^{-}{ }_{1}\right]\right\rangle$ in the interval representation. The truth values of big premises $\|a \rightarrow b\|=\left\langle i^{+}{ }_{1} ; i^{-}{ }_{1}\right\rangle \div\left\langle i^{+}{ }_{2} ; i^{-}{ }_{2}\right\rangle=\left\langle\left[i^{+}{ }_{1}, i^{+}{ }_{2}\right] ;\left[i^{-}{ }_{2} ; i^{-}{ }_{1}\right]\right\rangle$ (see figure 1). The $\bullet$ and $\oplus$ is t-norm and t-co-norm (s-norm) $[23,24]$ in infix notation with the additional property $1-x \bullet y=(1-x) \oplus(1-y)$ or $1-x \oplus y=(1-x) \bullet(1-y)$. The scheme for calculating the truth of the conclusion based on the truth of the premises is indicated after the colon. Expression (1) is a "modus ponens" inference rule for interval values of the truth vector.

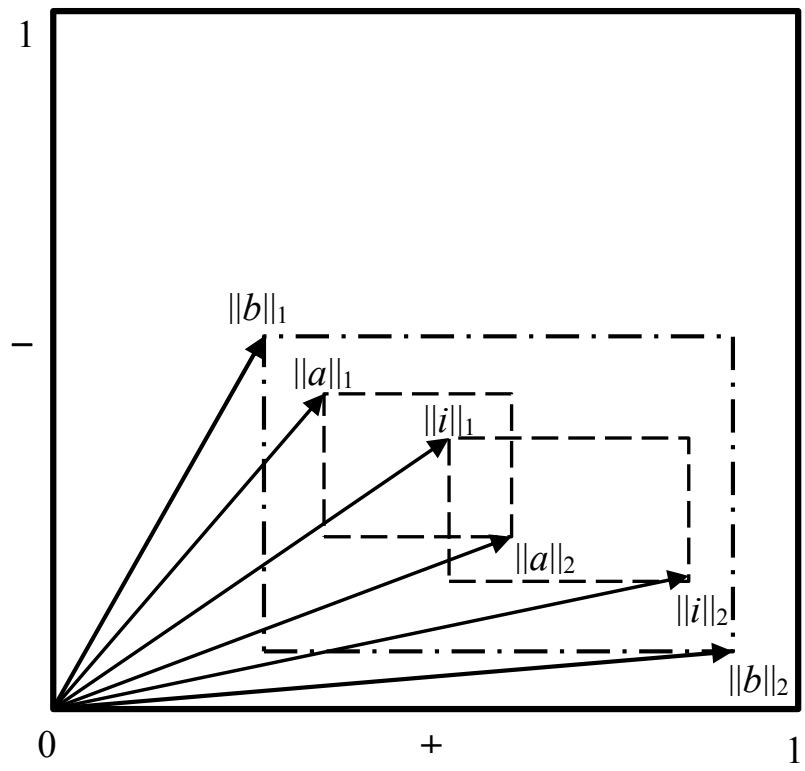

Figure 1. Illustration of the interval truth of premises and conclusions 


\section{Inference at abnormal big premises}

In real systems, the anomalous truth value of a big premise is not a real thing. It is unlikely that knowledge base developers will introduce false, uncertain, or contradictory knowledge into the system. Nevertheless, developers' mistakes are possible, so the following cases are considered.

\subsection{Premise is strict lie}

According to (1) with a strictly false large premise in the interval representation: $\|a \rightarrow b\|=\langle 0 ; 1\rangle \div\langle 0 ; 1\rangle=\langle[0,0] ;[1,1]\rangle$, the truth value of the conclusion falls in the area close to a strict lie:

$$
\|b\|=\left\langle a^{+}{ }_{1} \bullet 0 ; a^{-}{ }_{1} \oplus 1\right\rangle \div\left\langle a^{-}{ }_{1} \oplus 0 ; a^{+}{ }_{1} \bullet 1\right\rangle=\langle 0 ; 1\rangle \div\left\langle a^{-}{ }_{1} ; a^{+}{ }_{1}\right\rangle .
$$

Although this implication is an anomaly (a design error), other implications are assumed as strictly true. So the following implication $b \rightarrow c$ is strictly true. In the vector interval representation: $\|b \rightarrow c\|=\langle 1 ; 0\rangle \div\langle 1 ; 0\rangle=\langle[1,1] ;[0,0]\rangle$. Given this, as well as (1) and the properties of t-norms, one can write:

$$
\begin{gathered}
b, b \rightarrow c \mid-b:\|c\|=\left\langle b^{+}{ }_{1} \bullet i^{+}{ }_{1} ; b^{-}{ }_{1} \oplus i^{-}{ }_{1}\right\rangle \div\left\langle b^{-}{ }_{1} \oplus i^{+}{ }_{2} ; b^{+}{ }_{1} \bullet i^{-}{ }_{2}\right\rangle=\left\langle b^{+}{ }_{1} \bullet 1 ; b^{-}{ }_{1} \oplus 0\right\rangle \div\left\langle b^{-}{ }_{1} \oplus 1 ; b^{+}{ }_{1} \bullet 0\right\rangle= \\
=\left\langle b^{+}{ }_{1} ; b^{-}{ }_{1}\right\rangle \div\langle 1 ; 0\rangle=\left\langle\left[b^{+}{ }_{1}, 1\right] ;\left[0, b^{-}{ }_{1}\right]\right\rangle .
\end{gathered}
$$

Since, according to (2), $b^{+}{ }_{1}=0 ; b^{-}{ }_{1}=1$, finally find: $\|c\|=\langle[0,1] ;[0,1]\rangle$ (figure 2 ). This result is reproduced throughout the inference chain.

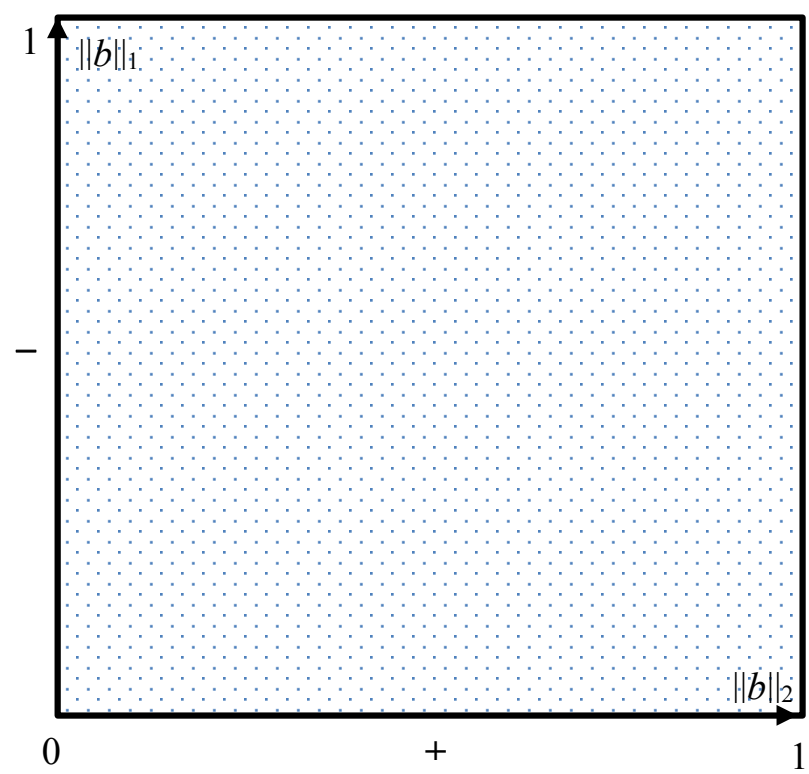

Figure 2. The truth area of the conclusion is Cartesian product $[0,1] \times[0,1]$

\subsection{Premise is uncertainty}

When there is uncertainty in the implication: $\|a \rightarrow b\|=\langle[0,0] ;[0,0]\rangle$, from (1) and the properties of tnorms, is obtained:

$$
\|b\|=\left\langle a^{+}{ }_{1} \bullet 0 ; a^{-} \oplus 0\right\rangle \div\left\langle a^{-}{ }_{1} \oplus 0 ; a^{+}{ }_{1} \bullet 0\right\rangle=\left\langle 0 ; a^{-}{ }_{1}\right\rangle \div\left\langle a^{-}{ }_{1} ; 0\right\rangle .
$$

In other words, the area of truth of the vector $\|b\|$ is a square with sides $\left[0, a^{-}{ }_{1}\right] \times\left[0, a^{-}{ }_{1}\right]$. If the small premise is strictly true or close to it, the uncertainty of the large premise generates the uncertainty of the 
conclusion (the vector $\|b\|$ is located near the point $\langle 0 ; 0\rangle$ ). If it is strictly false, one gets the already known square $[0,1] \times[0,1]$.

As in the previous case the next implication $b \rightarrow c$ regarding as true: $\|b \rightarrow c\|=\langle 1 ; 0\rangle \div\langle 1 ; 0\rangle=\langle[1,1] ;[0,0]\rangle$. Given this, as well as (1) and the properties of t-norms, one can write:

$$
\begin{gathered}
b, b \rightarrow c \mid-b:\|c\|=\left\langle b^{+}{ }_{1} \bullet i^{+}{ }_{1} ; b^{-}{ }_{1} \oplus i^{-}{ }_{1}\right\rangle \div\left\langle b^{-}{ }_{1} \oplus i^{+}{ }_{2} ; b^{+}{ }_{1} \bullet i^{-}{ }_{2}\right\rangle=\left\langle b^{+}{ }_{1} \bullet 1 ; b^{-}{ }_{1} \oplus 0\right\rangle \div\left\langle b^{-}{ }_{1} \oplus 1 ; b^{+}{ }_{1} \bullet 0\right\rangle= \\
=\left\langle b^{+}{ }_{1} ; b^{-}{ }_{1}\right\rangle \div\langle 1 ; 0\rangle=\left\langle\left[b^{+}{ }_{1}, 1\right] ;\left[0, b^{-}{ }_{1}\right]\right\rangle .
\end{gathered}
$$

Since, according to (3), $b^{+}{ }_{1}=0,{b^{-}}_{1}=a^{-}{ }_{1}$, the final result is: $\|c\|=\left\langle[0,1] ;\left[0, a^{-}{ }_{1}\right]\right\rangle$.

In the third step, assuming the implication $c \rightarrow d$ is also strictly true one gets:

$$
\begin{aligned}
c, c \rightarrow d \mid d:\|d\|= & \left\langle c^{+}{ }_{1} \bullet i^{+}{ }_{1} ; c^{-}{ }_{1} \oplus i^{-}{ }_{1}\right\rangle \div\left\langle c^{-}{ }_{1} \oplus i^{+}{ }_{2} ; c^{+}{ }_{1} \bullet i^{-}{ }_{2}\right\rangle=\left\langle c^{+}{ }_{1} \bullet 1 ; c^{-}{ }_{1} \oplus 0\right\rangle \div\left\langle c^{-}{ }_{1} \oplus 1 ; c^{+}{ }_{1} \bullet 0\right\rangle= \\
& =\left\langle c^{+}{ }_{1} ; c^{-}{ }_{1}\right\rangle \div\langle 1 ; 0\rangle=\left\langle 0 ; a^{-}{ }_{1}\right\rangle \div\langle 1 ; 0\rangle=\left\langle[0,1] ;\left[0, a^{-}{ }_{1}\right]\right\rangle .
\end{aligned}
$$

And so on. The result $\left\langle[0,1] ;\left[0, a_{1}^{-}\right]\right\rangle$(figure 3 ) is reproduced throughout the inference.

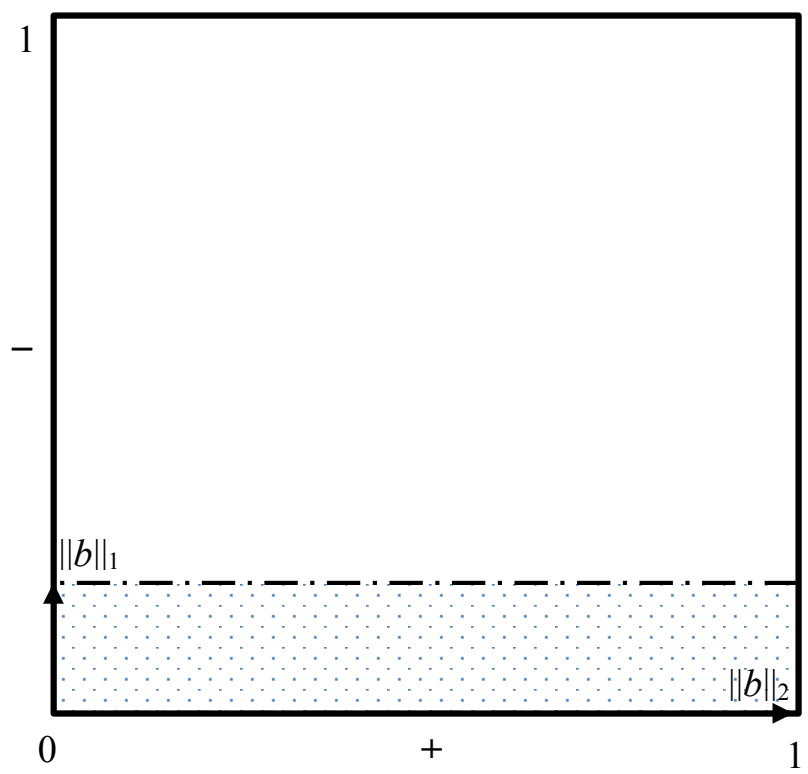

Figure 3. The truth area of the conclusion is $\left\langle[0,1] ;\left[0, a^{-}{ }_{1}\right]\right\rangle\left(\right.$ Cartesian product $\left.[0,1] \times\left[0, a^{-}{ }_{1}\right]\right)$

\subsection{Premise is complete contradiction}

If a big premise is complete contradiction: $\|b \rightarrow c\|=\langle[1,1] ;[1,1]\rangle$, from (1) follows:

$$
\|b\|=\left\langle a^{+}{ }_{1} \bullet 1 ; a^{-}{ }_{1} \oplus 1\right\rangle \div\left\langle a^{-}{ }_{1} \oplus 1 ; a^{+}{ }_{1} \bullet 1\right\rangle=\left\langle a^{+}{ }_{1} ; 1\right\rangle \div\left\langle 1 ; a^{+}{ }_{1}\right\rangle=\left\langle\left[a^{+}{ }_{1}, 1\right] ;\left[a^{+}{ }_{1}, 1\right]\right\rangle .
$$

That is, with a sufficiently reliable small premise, an area close to $\langle 1 ; 1\rangle$ is formed.

In the next step, if the next implication is strictly true, taking into account (4) one gets (figure 4):

$$
b, b \rightarrow c+b:\|c\|=\left\langle b^{+}{ }_{1} ; b^{-}{ }_{1}\right\rangle \div\langle 1 ; 0\rangle=\left\langle a^{+}{ }_{1} ; 1\right\rangle \div\langle 1 ; 0\rangle=\left\langle\left[a^{+}{ }_{1}, 1\right] ;[0,1]\right\rangle .
$$

And this set of values is also preserved throughout the inference. 


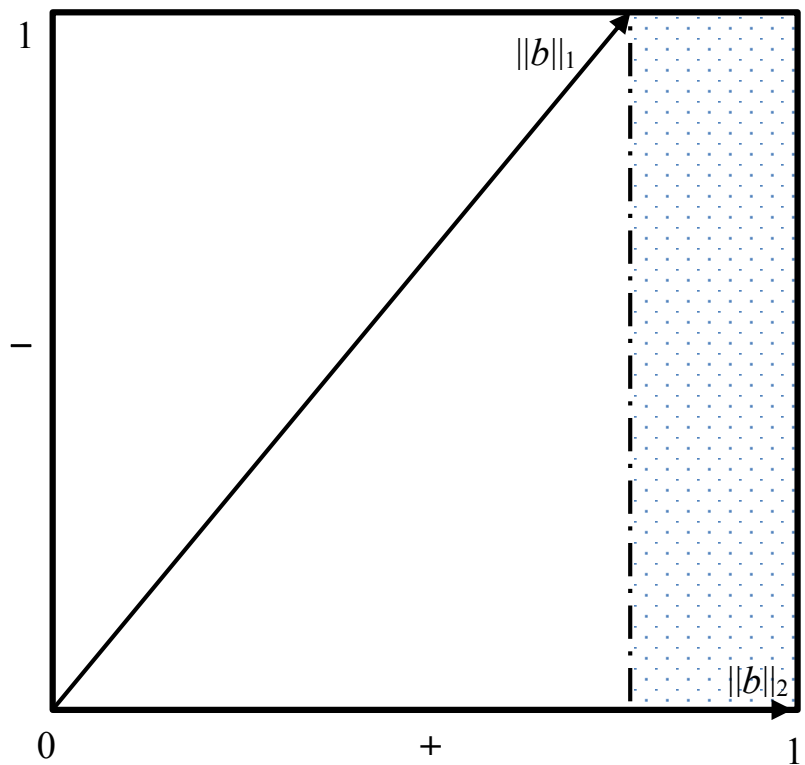

Figure 4. The truth area of the conclusion is $\left\langle\left[a^{+}, 1\right] ;[1,0]\right\rangle\left(\right.$ Cartesian product $\left.\left[a^{+}, 1\right] \times[1,0]\right)$

\section{Inference at abnormal small premises}

Anomalous truth values of small premises are more real. Anomalies may occur during inference due to hidden errors in the knowledge bases. As a result, in the intermediate stages of inference, the truth of a small premise may be poor.

\subsection{Premise is strictly lie}

Let strictly false premise appeared at some output step. As follows from (1) range of conclusion truth values becomes the square $[0,1] \times[0,1]$ at the first step. One should find out how this affects the next inference steps. Substituting $\|a\|=\langle 0 ; 0\rangle \div\langle 1 ; 0\rangle$ in the (1) and assuming the implication to be strictly true: $\|i\|=\langle 1 ; 0\rangle \div\langle 1 ; 0\rangle$, one gets $a^{+}{ }_{1}=0, a^{-}{ }_{1}=1, a^{+}{ }_{2}=1, a^{-}{ }_{2}=0, i^{+}{ }_{1}=1, i^{-}{ }_{1}=0, i^{+}{ }_{2}=1, i^{-}{ }_{2}=0$. As a result:

$$
\begin{gathered}
\|b\|=\left\langle a^{+}{ }_{1} \bullet i^{+}{ }_{1} ; a^{-}{ }_{1} \oplus i^{-}{ }_{1}\right\rangle \div\left\langle a^{-}{ }_{1} \oplus i^{+}{ }_{2} ; a^{+}{ }_{1} \bullet i^{-}{ }_{2}\right\rangle=\left\langle\left[a^{+}{ }_{1} \bullet i^{+}{ }_{1}, a^{-}{ }_{1} \oplus i^{+}{ }_{2}\right] ;\left[a^{+}{ }_{1} \bullet i^{-}{ }_{2}, a^{-}{ }_{1} \oplus i^{-}{ }_{1}\right]\right\rangle= \\
=\langle[0 \bullet 1,1 \oplus 1] ;[0 \bullet 0,1 \oplus 0]\rangle=\langle[0,1] ;[0,1]\rangle .
\end{gathered}
$$

That is, the truth of the conclusion belongs to the same square $[0,1] \times[0,1]$. Since truth of the antecedent belongs to the domain $[0,1] \times[0,1]$ and the same domain was obtained for the consequent, then in the third step and all subsequent steps it will be reproduced as well.

Introduce a measure of the accuracy of the judgment $b$ by the formula:

$$
\mu_{\mathrm{ToY}}(b)=1-\sqrt{\frac{\left(b_{2}^{+}-b_{1}^{+}\right)^{2}+\left(b_{2}^{-}-b_{1}^{-}\right)^{2}}{2}} ;
$$

(the precision is equal to one for the point values of the vector $\|b\|$ and zero when $\|b\| \in[0,1] \times[0,1]$; here $\left.\|b\|=\left\langle\left[b^{+}{ }_{1}, b^{+}{ }_{2}\right] ;\left[b^{-}{ }_{1}, b^{-}{ }_{2}\right]\right\rangle\right)$. Then it turns out that the zero accuracy of the conclusion, when the truth of the consequent $b$ is "anywhere" in the square $[0,1] \times[0,1]$, is reproduced at all steps of the conclusion, starting from the second. This is preserved until the terminal fact-hypothesis, signaling about artifact the falsity of the small premise at some of the inference steps.

Thus, the appearance of the anomaly of type strict falsity of small premises in the course of interval inference leads to the measure of the accuracy is zero that is a signal about the presence of an artifact. 


\subsection{Premise is uncertainty}

Next, we consider the case of uncertainty of a fact, when its truth vector has the value $\langle 0 ; 0\rangle$. As follows from (1), in this case, the small premise for the next output step takes an interval value:

$$
\|b\|=\left\langle 0 \bullet i^{+} ; 0 \oplus i^{-}\right\rangle \div\left\langle 0 \oplus i^{+} ; 0 \bullet i^{-}\right\rangle=\left\langle 0 ; i^{-}\right\rangle \div\left\langle i^{+} ; 0\right\rangle .
$$

Since all implications are declared strictly true, this gives:

$$
\|b\|=\langle 0 \bullet 1 ; 0 \oplus 0\rangle \div\langle 0 \oplus 1 ; 0 \bullet 0\rangle=\langle 0 ; 0\rangle \div\langle 1 ; 0\rangle .
$$

That is, $b^{+}{ }_{1}=0, b^{-}{ }_{1}=0, b^{+}{ }_{2}=1, b_{2}^{-}=0$. Since $b$ is the premise for the second step, then still assuming $i^{+}{ }_{1}=1, i^{-}{ }_{1}=0, i^{+}{ }_{2}=1, i^{-}{ }_{2}=0$, for the conclusion $c$ we find (figure 5):

$$
\begin{gathered}
\|c\|=\left\langle b^{+}{ }_{1} \bullet i^{+}{ }_{1} ; b^{-}{ }_{1} \oplus i^{-}{ }_{1}\right\rangle \div\left\langle b^{-}{ }_{1} \oplus i^{+}{ }_{2} ; b^{+}{ }_{1} \bullet i^{-}{ }_{2}\right\rangle=\left\langle\left[b^{+}{ }_{1} \bullet i^{+}{ }_{1}, b^{-}{ }_{1} \oplus i^{+}{ }_{2}\right] ;\left[b^{+}{ }_{1} \bullet i^{-}{ }_{2}, b^{-}{ }_{1} \oplus i^{-}{ }_{1}\right]\right\rangle= \\
=\langle[0 \bullet 1,0 \oplus 1] ;[0 \bullet 0,0 \oplus 0]\rangle=\langle[0,1] ;[0,0]\rangle .
\end{gathered}
$$

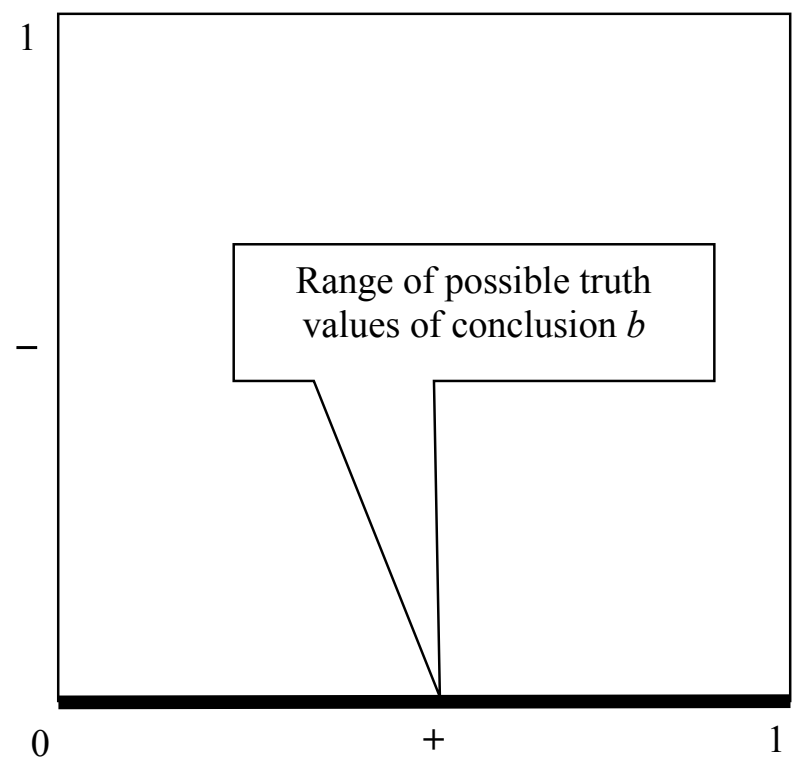

Figure 5. The range of possible truth values of conclusion $b$ according to (5)

The truth of the conclusion falls back into the range $\langle 0 ; 0\rangle \div\langle 1 ; 0\rangle$ (or $\langle[0,1] ;[0,0]\rangle$ in the interval representation). Obviously all subsequent steps will give the same result.

Thus, the appearance of an anomaly of the type uncertainty of a small premise at the start or during the output with interval truth values leads to an anomalous truth value that is reproduced along the chain $\langle[0,1] ;[0,0]\rangle$, which is a signal about the presence of the corresponding artifact.

\subsection{Premise is complete contradiction}

Finally, in the case of a complete contradiction, when the starting or intermediate small premise takes the truth value $\|a\|=\langle 1 ; 1\rangle$ and large premise is strictly true, the corresponding inference step generates the truth of the conclusion $\|b\|=\langle 1 ; 1\rangle \div\langle 1 ; 0\rangle=\langle[1,1] ;[0,1]\rangle$. Accordingly, when $b$ enters the next rule, one gets (figure 6):

$$
\begin{gathered}
\|c\|=\left\langle b^{+}{ }_{1} \bullet i^{+}{ }_{1} ; b^{-}{ }_{1} \oplus i^{-}{ }_{1}\right\rangle \div\left\langle b^{-}{ }_{1} \oplus i^{+}{ }_{2} ; b^{+}{ }_{1} \bullet i^{-}{ }_{2}\right\rangle=\left\langle\left[b^{+}{ }_{1} \bullet i^{+}{ }_{1}, b^{-}{ }_{1} \oplus i^{+}{ }_{2}\right] ;\left[b^{+}{ }_{1} \bullet i^{-}{ }_{2}, b^{-}{ }_{1} \oplus i^{-}{ }_{1}\right]\right\rangle= \\
=\langle[1 \bullet 1,1 \oplus 1] ;[1 \bullet 0,1 \oplus 0]\rangle=\langle[1,1] ;[0,1]\rangle .
\end{gathered}
$$




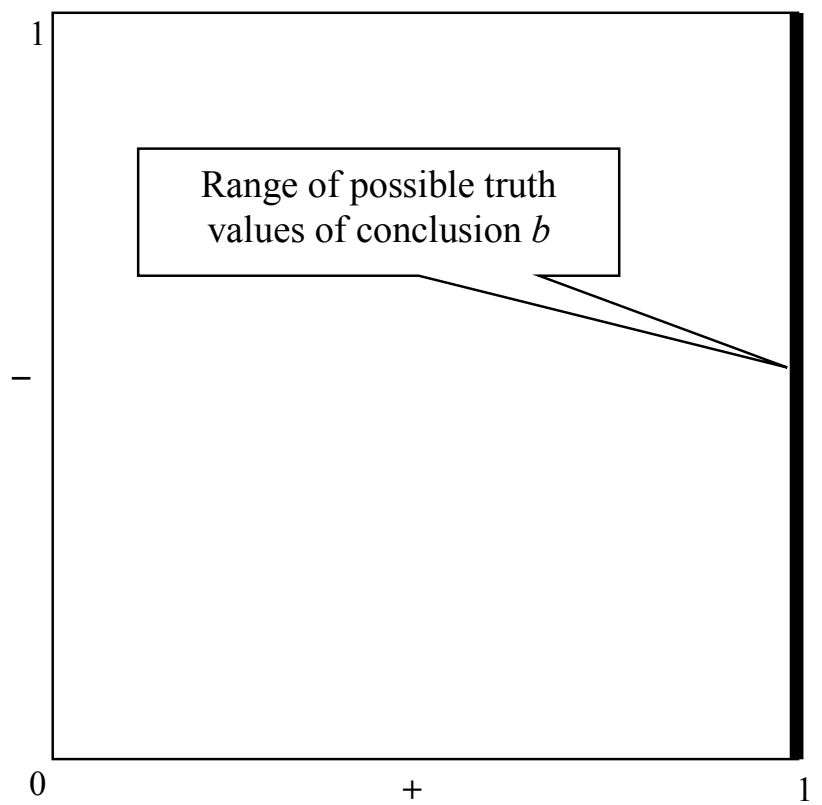

Figure 6. The range of possible truth values of conclusion $b$ according to (6)

That is, the truth of the antecedent $\langle[1,1] ;[0,1]\rangle$ leads to the truth of the consequent as well $\langle[1,1] ;[0,1]\rangle$. As in the case of uncertainty, this means that a complete contradiction generates a chain of the same truth values $\langle[1,1] ;[0,1]\rangle$. And here the anomaly reproduces an anomaly of the same type.

Thus the appearance of an anomaly such as a complete contradiction of a small premise at the start or during the inference leads to an anomalous truth value being reproduced along the chain $\langle[1,1] ;[0,1]\rangle$, it signals the presence of the corresponding artifact.

\section{Example}

Suppose there is a knowledge base with rules:

$$
\begin{gathered}
a \rightarrow b ; \\
a \rightarrow \neg b ; \\
b \rightarrow c ; \\
c \rightarrow d .
\end{gathered}
$$

Rules are ordered in a causal manner. That is, the rule containing the fact $f$ on the right always precedes the rule containing $f$ on the left (this is easy to do). All the rules are strictly true. Their truth is equal to $\langle[1,1] ;[0,0]\rangle$ in interval representation:

$$
\|a \rightarrow b\|=\left\|a^{\prime} \rightarrow \neg b\right\|=\|b \rightarrow c\|=\|c \rightarrow d\|=\langle[1,1] ;[0,0]\rangle .
$$

It is assumed that the answer "Yes" to questions $a$ and $a$ ' is admissible and the user (or the verifying component) has chosen it. It is in interval notation

$$
\|a\|=\left\|a^{\prime}\right\|=\langle[1,1] ;[0,0]\rangle .
$$

According to (1), (11), (12), the first inference step will be:

$a, a \rightarrow b \vdash b:\|b\|=\left\langle\left[a^{+}{ }_{1} \bullet i^{+}{ }_{1}, a^{-}{ }_{1} \oplus i^{+}{ }_{2}\right] ;\left[a^{+}{ }_{1} \bullet i^{-}{ }_{2}, a^{-}{ }_{1} \oplus i^{-}{ }_{1}\right]\right\rangle=\langle[1 \bullet 1,0 \oplus 1] ;[1 \bullet 0,0 \oplus 0]\rangle=\langle[1,1] ;[0,0]\rangle$. 
Further, the second step of the inference would be:

$$
a^{\prime}, a^{\prime} \rightarrow \neg b \vdash \neg b:\|\neg b\|=\langle[1,1] ;[0,0]\rangle .
$$

This means $\|b\|$ is equal to $\langle[0,0] ;[1,1]\rangle$. Therefore:

$$
\begin{aligned}
\|b\| & =\|b\|_{1}=\langle[1,1] ;[0,0]\rangle ; \\
\|b\| & =\|b\|_{2}=\langle[0,0] ;[1,1]\rangle .
\end{aligned}
$$

Combining of evidence is performed in the third step according to the rule [11, 12]:

If the truth vector $\|b\|$ has two meanings:

$$
\begin{gathered}
\|b\|_{1}=\left\langle\left[b^{+}{ }_{11}, b^{+}{ }_{12}\right] ;\left[b^{-}{ }_{12}, b^{-}{ }_{11}\right]\right\rangle ; \\
\|b\|_{2}=\left\langle\left[b^{+}{ }_{21}, b^{+}{ }_{22}\right] ;\left[b^{-}{ }_{22}, b^{-}{ }_{21}\right]\right\rangle ; \\
\text { then the combined truth value of b is: } \\
\|b\|=\left\langle\left[b^{+}{ }_{11} \oplus b^{+}{ }_{21}, b^{+}{ }_{12} \oplus{b^{+}}^{+} 2\right] ;\left[b^{-}{ }_{12} \oplus{b^{-}}^{-} 2, b^{-}{ }_{11} \oplus{b^{-}}{ }_{21}\right]\right\rangle .
\end{gathered}
$$

In the considered example, it turns out:

$$
\|b\|=\langle[1,1] ;[1,1]\rangle .
$$

This is a complete contradiction.

The anomalous truth value is obtained at the fourth step according to (1), (13):

$$
b, b \rightarrow c \mid c:\|c\|=\left\langle\left[b^{+}{ }_{1} \bullet i^{+}{ }_{1}, b^{-}{ }_{1} \oplus i^{+}{ }_{2}\right] ;\left[b^{+}{ }_{1} \bullet i^{-}{ }_{2}, b^{-}{ }_{1} \oplus i^{-}{ }_{1}\right]\right\rangle=\langle[1 \bullet 1,1 \oplus 1] ;[1 \bullet 0,1 \oplus 0]\rangle=\langle[1,1] ;[0,1]\rangle .
$$

The same value is obtained in the fifth step:

$$
c, c \rightarrow d \vdash d:\|d\|=\left\langle\left[c^{+}{ }_{1} \bullet i^{+}{ }_{1}, c^{-}{ }_{1} \oplus i^{+}{ }_{2}\right] ;\left[c^{+}{ }_{1} \bullet i^{-}{ }_{2}, c^{-}{ }_{1} \oplus i^{-}{ }_{1}\right]\right\rangle=\langle[1 \bullet 1,1 \oplus 1] ;[1 \bullet 0,1 \oplus 0]\rangle=\langle[1,1] ;[0,1]\rangle .
$$

The $c$ is final hypothesis. The source of the anomaly is found by backtracking. These are the rules (7) and (8).

This type of inference is implemented in the software system for modeling plausible reasoning "Heraclitus" developed by the authors.

\section{Conclusion}

Thus, one could conclude the following. When using $\mathrm{V}^{\mathrm{TF}}$-logics with interval values of the truth vector:

1. The presence of knowledge base artifacts that lead to strictly false, uncertain or contradictory assumptions in the inference generates appropriate and rather specific values of the truth of the conclusion.

2. These values are subsequently stored throughout the inference up to the final hypothesis, it is a signal of the presence of the corresponding artifact.

3. This feature can be used to detect knowledge base artifacts during dynamic verification.

\section{References}

[1] Artificial intelligence and expert systems: knowledge-based systems. - https://www.vfu.bg/en/eLearning/Artificial-Intelligence--AI_and_ES_Nowledge_base_systems.pdf.

[2] Brown C E and O'Leary D E 1995 Introduction to artificial intelligence and expert systems. https://msbfile03.usc.edu/digitalmeasures/doleary/intellcont/Brown-Oleary-es_tutor-1.htm

[3] Artificial Intelligent. Models and Methods: handbook 1999 ed D A Pospelov (Moscow: Radio and Communications) p 304 (in Russian)

[4] Jiarratano J C, Riley G 2005 Expert Systems: Principles and Programming, $4^{\text {th }}$ edn. (Course Technology).

[5] Gochet P, Gregoire E, Gribomont P, etc. 1988 From standard logic to logic programming: introducing a logic based approach to artificial intelligence (John Wiley \& Sons) 
[6] Shortliffe E H and Buchanan B G 1975 A model of inexact reasoning in medicine Mathematical Bioscience 23 pp 351-379.

[7] Shortliffe E H 1976 Computer-Based Medical Consultation: MYCIN (N.Y.: American Elsevier)

[8] Quinlan J R 1983 INFERNO: a causations approach to uncertain inference The computer J. 26(3) pp 255-269.

[9] Shafer G 1976 A Mathematical Theory of Evidence (Princeton and London: Princeton University Press) p 297

[10] Arshinskiy L V 1998 Methods of processing of nonstrict propositions] (Irkutsk: East-Siberian Institute of MIA of Russia) p 40 (in Russian)

[11] Arshinskiy L V 2005 Interval truth estimation in automated reasoning systems based on $\mathrm{V}^{\mathrm{TF}}$-logic SICPRO'05 (Electronic Materials) (Moscow: IPU RAN n/a V.A. Trapeznikov) pp 1061-1074 (in Russian)

[12] Arshinskiy L V 2007 Vector logic: foundations, concepts, models (Irkutsk: Irkutsk state university) 228 p. (In Russian).

[13] Arshinskii L V 2007 Substantial and Formal Deductions in Logics with Vector Semantics, Automation and Remote Control. 68(1) pp 139-148

[14] Tepandi J 1990 Comparison of Expert System Verification Criteria: Redundancy Proc. ECAI 90 Conf. Stockholm pp 49-62

[15] Zhang D 1993 Knowledge Base Verification: Issues and Approaches AAAI Technical Report WS-93-05 pp 148-49

[16] Antoniou G, van Harmelen F, Plant R and Vanthinen J 1998 Verification and Validation of Knowledge-Based Systems AI Magazine vol 19 no 3 (Workshop Report) pp 123-26

[17] Pospelova L Y and Chukanova O V 2009 The search for contradictions in the production knowledge bases Information and telecommunication systems 5 (Moscow: National Research Nuclear University) pp 23-27 (in Russian)

[18] Dolinyna O N 2010 Classification of errors in the knowledge bases of expert systems Bulletin of SSTU no 4(50) issue 2 pp 125-30 (in Russian)

[19] Proskuriakov D P 2015 Conflict resolution management in rule-based expert systems Proceedings of Irkutsk State Technical University no 8(103) pp 47-51 (in Russian)

[20] Sun Y, Wu T, Li X and Guizani M, 2017 A Rule Verification System for Smart Buildings IEEE Transactions on Emerging Topics in Computing 5 no 3 pp 367-79

[21] Arshinskiy L V, Ermakov A A and Nitezhuk M S 2019 Logic with Vector Semantic as a Means of Knowledge Bases Verification Ontology of designing 9(4) pp 510-521 DOI: 10.18287/2223-9537-2019-9-4-510-521 (in Russian)

[22] Arshinskiy L V, Ermakov A A and Nitezhuk M S 2020 Complex verification of rule-based knowledge bases using $\mathrm{V}^{\mathrm{TF}}$-logic Ontology of designing 10(1) pp 112-120 DOI: 10.26731/2658-3704.2020.3(8).61-70 (in Russian)

[23] Menger K 1942 Statistical metrics Proc. Nat. Acad. Sci. USA 8 pp 535-537

[24] Gottwald S 2000 Treatise on Many-Valued Logics (Leipzig) 604 p. 